\title{
Editorial
}

\section{Advanced Antenna Technologies in the Beyond IMT-Advanced Systems}

\author{
Mugen Peng, ${ }^{1}$ Cheng-Xiang Wang, ${ }^{2}$ Feifei Gao, ${ }^{3}$ and Wei Xiang ${ }^{4}$ \\ ${ }^{1}$ The Key Laboratory of Universal Wireless Communications for the Ministry of Education, \\ Beijing University of Posts and Telecommunications, Beijing 100876, China \\ ${ }^{2}$ School of Engineering \& Physical Sciences, Heriot-Watt University, Edinburgh EH14 4AS, UK \\ ${ }^{3}$ Department of Automation, Tsinghua University, Beijing 100084, China \\ ${ }^{4}$ School of Mechanical and Electrical Engineering, University of Southern Queensland (USQ), \\ Toowoomba, QLD 4350, Australia
}

Correspondence should be addressed to Mugen Peng; pmg@bupt.edu.cn

Received 2 October 2013; Accepted 2 October 2013

Copyright (C) 2013 Mugen Peng et al. This is an open access article distributed under the Creative Commons Attribution License, which permits unrestricted use, distribution, and reproduction in any medium, provided the original work is properly cited.

Both the fourth generation (4G) and fifth generation (5G) mobile systems will use multiple-input multiple-output (MIMO) technologies with multiantenna capabilities at both the base station and user equipment. To improve the capacity, extend the coverage, and decrease the antenna deployment complexity, many advanced antenna technologies have been proposed recently, such as the large-scale array antenna and the small-scale smart antenna. The large-scale array antenna system, also known as massive MIMO or massive threedimension (3D) antenna, is a new research field in antenna systems, electronics, communication theory, and embedded software. It is often utilized in the frequency division duplex(FDD-) based 4G \& 5G systems, and its performance gain over the classical array antenna, which uses a relatively small number of antennas fed via coaxial cables from a highpower amplifier, is thus significant. However, one of the disadvantages of massive MIMO systems is that the antenna size is too large, resulting in a large frontal area. Meanwhile, the miniaturization of smart antenna (also known as the small-scale smart antenna) is often designed for the time division duplex (TDD) 4G \& 5G systems and can effectively decrease the antenna size, while ensuring the capacity and coverage performances.

To fully realize the potential of advanced antenna technologies and to solve the problems of antenna application, this special issue aims to attract the attention of the research community for developing advanced and innovative antenna-related methodologies and techniques. We have received a total of 18 original submissions, out of which 8 papers were accepted for publication after peer reviewing. We regret that we had to reject many good papers due to the limited number of papers that can be published within this special issue. The accepted papers cover a broad area of advanced antenna topics.

Propagation and antenna models are indispensable for investigating advanced antenna techniques. A paper entitled "Full-wave analysis of microstrip antennas in a three-layered spherical media" by C. Yin et al. proposes an antenna model and the dyadic Green's function in spherical microstrip antennas. Furthermore, the asymptotic extraction approach is presented to accelerate the Green's functions convergence speed when source and field points locate in the same layer and different layers.

In a paper entitled "Dynamic beamforming for three dimensional MIMO technique in LTE-advanced networks" by $\mathrm{Y}$. Li et al. the authors present a dynamic beamforming algorithm where vertical domain of antenna is fully considered and beamforming vector can be obtained according to UEs' horizontal and vertical directions. At most 34\% gain for cell center UE and $92 \%$ gain for cell edge UE over the conventional 2D beamforming algorithm are achieved by the proposed algorithm. Another paper entitled "Limited feedback precoding for massive MIMO" by X. Su et al. proposes an avenue of codebook design based on a Kroneckertype approximation of the array correlation structure for the uniform rectangular antenna array. Simulation results 
demonstrate that the proposed codebook outperforms the exisiting codebooks remarkably.

A paper entitled "The study of indoor and field trials on $2 \times 8$ MIMO architecture in TD-LTE network" by X. Zhang et al. analyzes measurement methods of four MIMO transmission modes (TMs) in time division-LTE (TD-LTE), where indoor and field tests are presented to investigate how real-world propagation affects the capacity and the error performance of MIMO transmission scheme. In the fifth paper entitled "Energy efficiency maximization through cooperative transmit and receive antenna selection for multicell MU-MIMO system" by Y. Dong et al., an antenna selection scheme which can maximize the energy efficiency of BS cluster is proposed, and a cost function varying with the number of antennas is presented. Also, the effect of the antenna selection scheme on the energy efficiency of both the serving BS and the neighbor BS is investigated.

For the advanced relevant wireless communication techniques, a paper entitled "Interference coordination in multiple antenna based LTE-advanced heterogeneous systems" by Y. Li et al. presents two interference coordination (IC) schemes based on whether the receiver coding matrix is full rank or not, named as full coding (IC-FC) and part coding (IC$\mathrm{PC})$, respectively. A paper entitled "Power scaling for spatial modulation with limited feedback" by Y. Xiao proposes the power scaling spatial modulation (PS-SM) scheme. The main idea is based on the introduction of a scaling factor (SF) for weighting the modulated symbols on each transmit antenna of SM, so as to enlarge the minimum Euclidean distance of modulated constellations and improve the system performance. In another paper entitled "Secrecy balancing over two-user MISO interference channels with rician fading" by J. $\mathrm{Ni}$ considers a 2-user multiple-input single-output (MISO) interference channel with confidential messages (IFC-CM) in which a quadratic relationship between the real-valued parameters of two users are derived for the Rician channel to reach the ergodic secrecy rate balancing point.

\section{Acknowledgment}

Before the end of this editorial, the authors would like to thank the anonymous reviewers for their great efforts in reviewing the submitted papers, without which this special issue would not have been published as scheduled.

$$
\begin{array}{r}
\text { Mugen Peng } \\
\text { Cheng-Xiang Wang } \\
\text { Feifei Gao } \\
\text { Wei Xiang }
\end{array}
$$



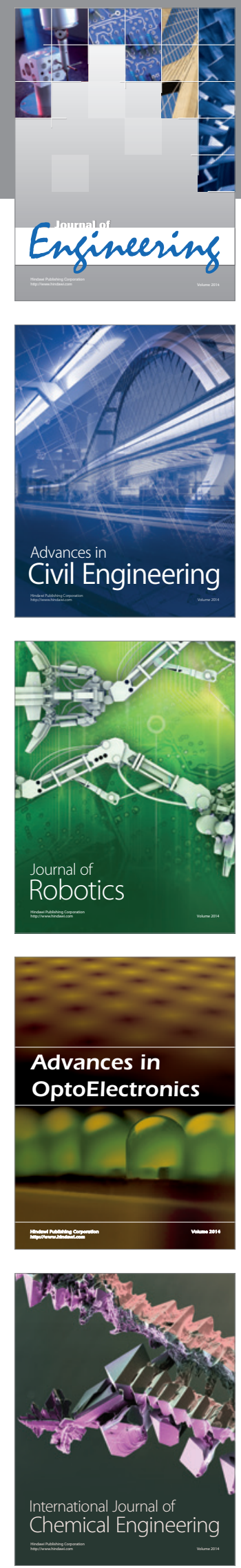

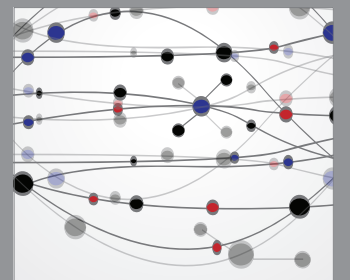

The Scientific World Journal
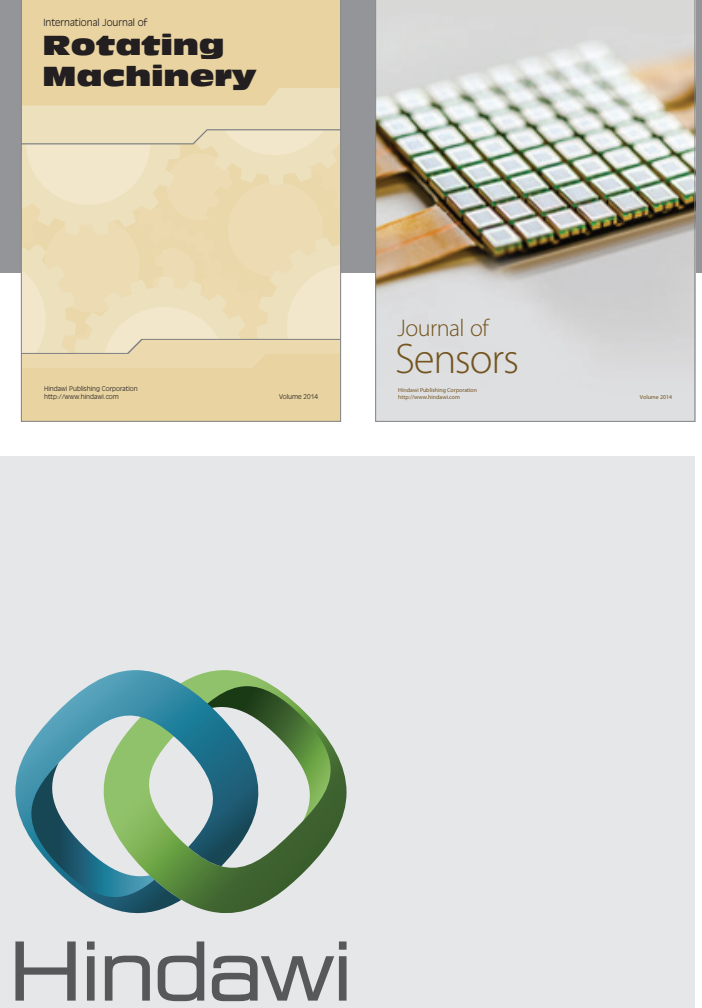

Submit your manuscripts at http://www.hindawi.com
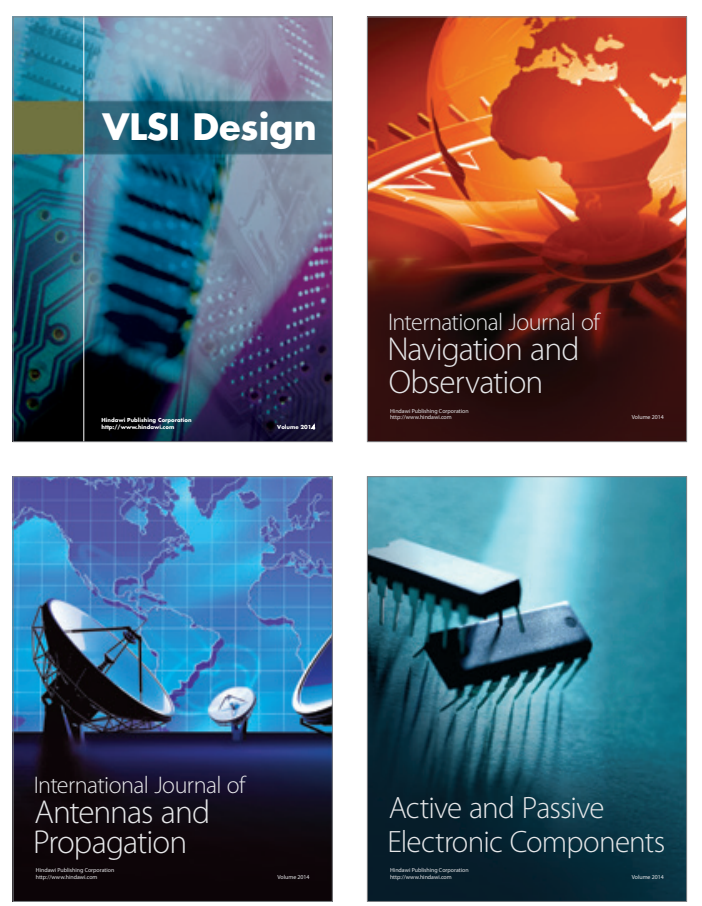
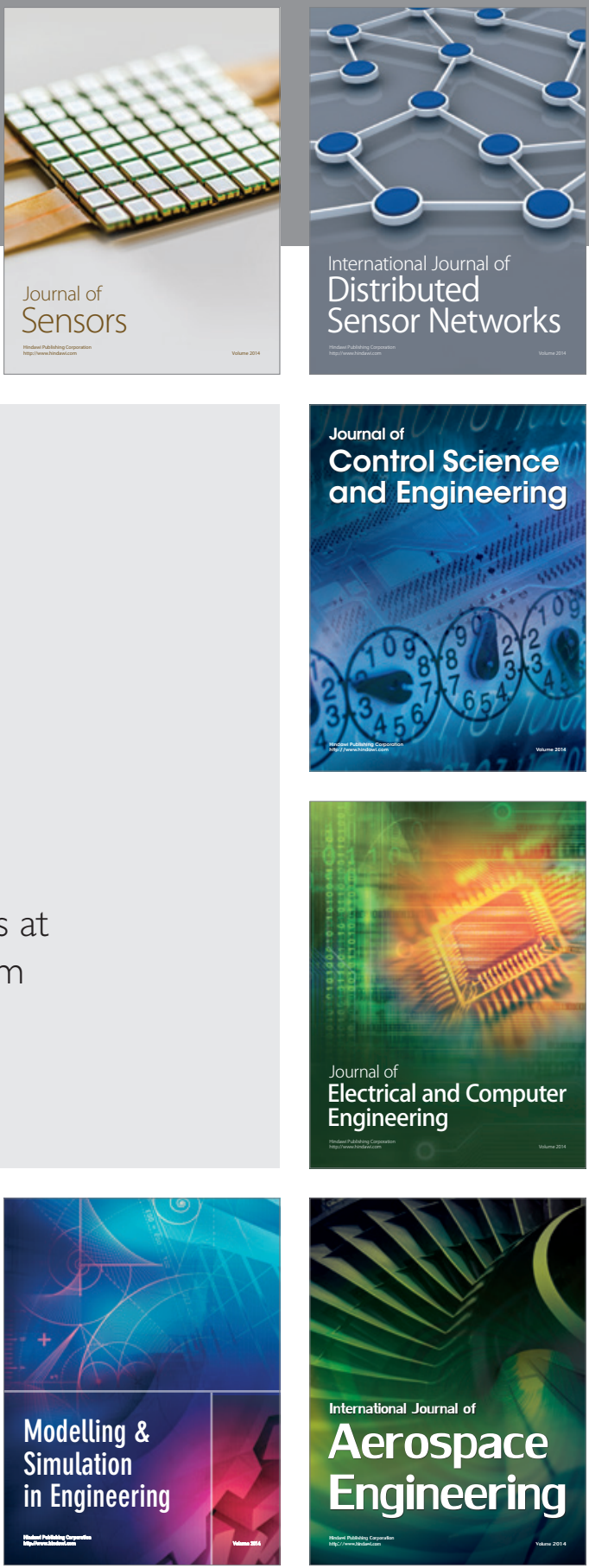

Journal of

Control Science

and Engineering
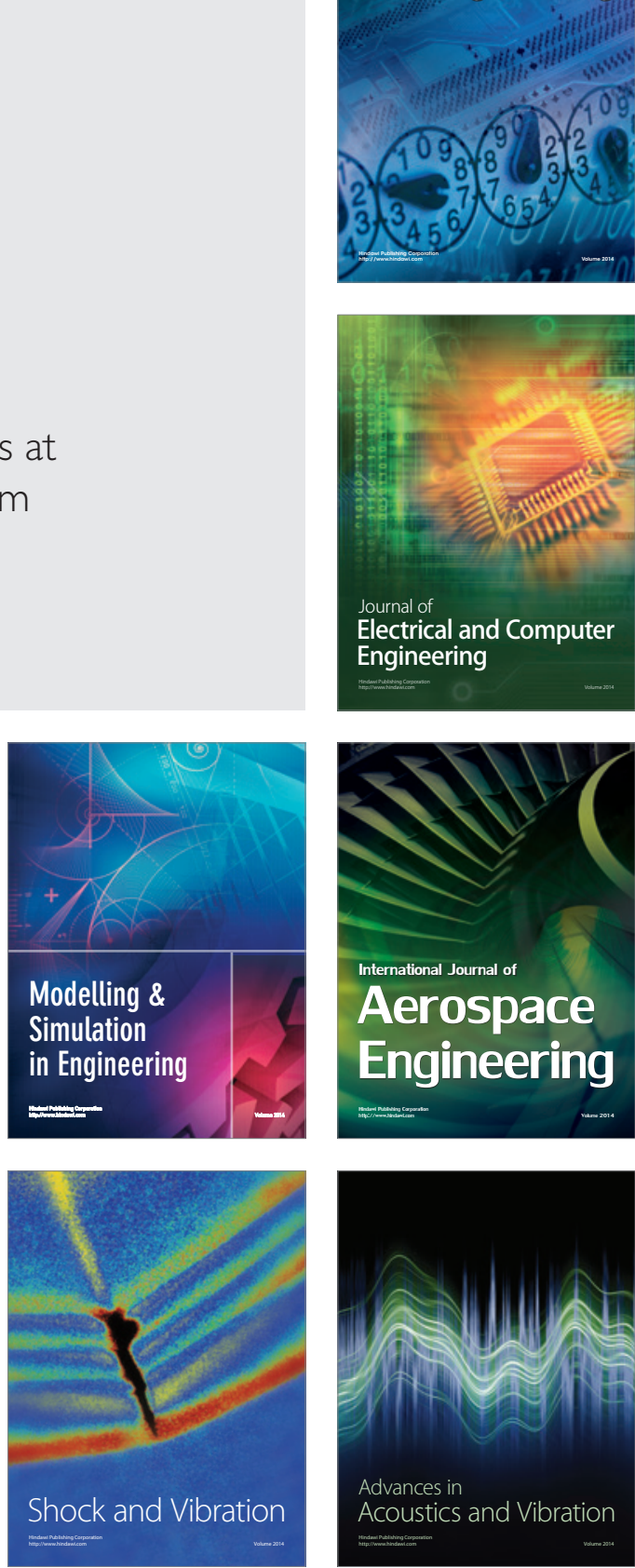